\section{Efeitos do clampeamento tardio do cordão umbilical sobre os níveis de hemoglobina e ferritina em lactentes aos três meses de vida}

\author{
Effects of delayed cord clamping on hemoglobin \\ and ferritin levels in infants at three months of age
}

\footnotetext{
${ }^{1}$ Instituto de Saúde, Secretaria de Estado da Saúde de São Paulo, São Paulo, Brasil. 2 Hospital Municipal Fernando Mauro Pires da Rocha, São Paulo, Brasil.

Correspondência S. I. Venânico Instituto de Saúde, Secretaria de Estado da Saúde de São Paulo.

Rua Santo Antonio 590, $3^{\circ}$ andar, São Paulo, SP 01314-000, Brasil. soniav@isaude.sp.gov.br
}

\begin{abstract}
This study assessed the effect of delayed (1 minute after delivery) clamping of the umbilical cord on hemoglobin and ferritin levels in infants at three months of age. Mothers and their infants born through vaginal delivery, at term, and without congenital anomalies (325 pairs) were recruited at a hospital in São Paulo, Brazil, in 2006 (164 in the delayed clamping subgroup and 161 in the early clamping subgroup). Maternal hemoglobin at delivery, umbilical cord hemoglobin, and ferritin were recorded. At three months follow-up, venous blood samples were drawn from 224 (69\%) infants for hemoglobin and ferritin measurement. Socioeconomic, maternal reproductive, anthropometric, and infant feeding variables were studied. Multiple linear regression models were used to analyze the data. The effect of delayed clamping at birth, measured at three months, was only significant for ferritin ( $p=0.040)$, and the concentration was higher $(23.29 \mathrm{ng} / \mathrm{mL})$ in this subgroup as compared to the early clamping subgroup. Delayed umbilical cord clamping can serve as a strategy to improve infant iron status and prevent iron deficiency.
\end{abstract}

Nutritional Epidemiology; Anemia; Iron Deficiency; Child; Umbilical Cord
Sonia Isoyama Venâncio ${ }^{1}$

Renata Bertazzi Levy ${ }^{1}$

Sílvia Regina Dias Médici Saldiva ${ }^{1}$

Lenise Mondini 1

Maria Cecília Goi Porto Alves 1

Siu Lum Leung 2

\section{Introdução}

A deficiência de ferro e a anemia constituem a carência nutricional mais severa e freqüente no mundo ${ }^{1}$. Além de afetar um número elevado de crianças e mulheres em países em desenvolvimento, é a única carência que também atinge estes grupos populacionais em países desenvolvidos. Depois das gestantes, as crianças menores de dois anos são as mais atingidas $1,2,3$.

Segundo a Organização Mundial da Saúde, em 150 países membros, de um total de 197, a anemia é considerada problema moderado ou grave de saúde pública, sendo que $47,4 \%$ das crianças menores de cinco anos e $41,8 \%$ das gestantes são acometidas pelo agravo em todo o mundo. Na América Latina, a prevalência entre os pré-escolares é de 39,5\% e entre as gestantes, de $31,1 \% 4$.

No Brasil, estima-se que aproximadamente 5 milhões de crianças menores de quatro anos sofram de anemia 5 . Na década de 90 cinco inquéritos de base populacional foram realizados em centros urbanos das regiões Nordeste, Sudeste e Sul do país e revelaram altas prevalências de anemia em crianças menores de cinco anos:45,2\% no Município deSãoPaulo6;39,6\% na Região Metropolitana do Recife, Pernambuco 7; 47,8\% em Porto Alegre, Rio Grande do Sul 8; e $54 \%$ em Criciúma, Santa Catarina 9. No Município de São Paulo, que conta com inquéritos anteriores, observa-se tendência de aumento 
da prevalência de anemia nas últimas décadas, passando de $23,1 \%$ em $1973 / 1974$ para $35,5 \%$ em 1984/1985 e para 46,9\% em 1995/1996.

Em crianças a anemia pode provocar diminuição da capacidade cognitiva, distúrbios comportamentais, falta de memória, baixa concentração mental, déficit de crescimento, diminuição da força muscular e da atividade física, além de maior suscetibilidade a doenças infecciosas 2,3 .

Embora vários fatores possam levar à anemia, admite-se que a causa principal, responsável pelas elevadas prevalências da enfermidade na infância, seja uma dieta com pouca quantidade de ferro ou com ferro de baixa biodisponibilidade 10 .

Como a alimentação vem sendo apontada como principal fator associado à deficiência de ferro e anemia, as intervenções mais freqüentes para seu controle são a suplementação medicamentosa e a fortificação de alimentos. Ambas vêm tendo sucessos pontuais, porém demandam custo elevado e logística complexa para diminuição da prevalência global, principalmente em países em desenvolvimento.

Além da dieta, a deficiência materna do mineral, durante a gestação, pode acarretar redução do estoque de ferro do recém-nascido, uma vez que não há transferência adequada através da placenta, predispondo a criança à anemia 11 . Outros fatores potencialmente de risco para a deficiência de ferro, apontados na literatura, são o baixo peso ao nascer e o sexo masculino 1,12 .

Sabe-se também que os níveis de ferro na criança são fortemente influenciados pelo volume corpóreo total de ferro ao nascimento. As práticas obstétricas, particularmente o momento do clampeamento do cordão umbilical, podem afetar o volume de sangue transferido da placenta para o recém-nascido e conseqüentemente o volume total de ferro 13.

Van Rheenen \& Brabin 13,14, em revisão sistemática, apontam evidências de que o clampeamento tardio comparado ao clampeamento imediato está associado com maior concentração de hemoglobina e menor incidência de anemia aos quatro meses de vida e estoques mais elevados de ferro aos seis meses.

Considerando a alta prevalência de anemia infantil e materna no país e a inexistência de estudos nacionais que avaliem o impacto do tempo de clampeamento do cordão umbilical, o presente trabalho teve por objetivo avaliar os efeitos do clampeamento tardio sobre os níveis de hemoglobina e ferritina em lactentes aos três meses de vida.

\section{Metodologia}

O estudo foi realizado no Hospital Municipal do Campo Limpo, São Paulo, Brasil, entre abril de 2006 e março de 2007. A maternidade realizava em torno de 300 partos/mês, adotando como rotina o clampeamento precoce do cordão umbilical, ou seja, imediatamente após o nascimento do bebê.

Realizou-se um estudo de intervenção, no qual as crianças foram alocadas em dois grupos: o do clampeamento imediato e o do clampeamento tardio. Posteriormente, foram avaliadas aos três e seis meses após o nascimento quanto aos níveis de ferritina e hemoglobina. A intervenção caracterizou-se pela realização do clampeamento do cordão umbilical um minuto após o nascimento com manutenção do recém-nascido no mesmo nível da placenta. A alocação em grupos foi realizada, por questões operacionais, pela alteração semanal do procedimento (clampeamento imediato ou clampeamento tardio), sendo que na primeira semana o procedimento sorteado foi o do clampeamento tardio.

Foram incluídas no estudo crianças nascidas a termo, de parto normal e sem patologias. Consideraram-se critérios de exclusão: patologias gestacionais, como eclampsia, pré eclampsia, diabetes, isoimunização RH, sendo também excluídos os casos de anóxia neonatal detectados no momento do parto.

O tamanho da amostra foi calculado considerando-se o objetivo de estimar o risco relativo da ocorrência de anemia em crianças com clampeamento imediato em relação àquelas com clampeamento tardio. Utilizando-se o programa Epi Info (aplicativo Epitable; Centers for Disease Control and Prevention, Atlanta, Estados Unidos), determinou-se um risco relativo de 1,5 a ser detectado, com erros do tipo I e II de 0,05 e 0,10, respectivamente, e prevalência de anemia de 0,30 entre as crianças com clampeamento imediato e estimou-se o tamanho da amostra em 280 crianças. Prevendo 13\% de perdas no seguimento, foi planejada a inclusão de 322 crianças no estudo.

A captação das crianças ocorreu entre abril e dezembro de 2006. Estimou-se inicialmente que a duração da captação seria de três meses. Porém, houve pouca adesão da equipe do hospital aos procedimentos previstos para a inclusão das mães no estudo. Nesse período foram realizados 1.499 partos vaginais, dos quais 325 foram captados seguindo os critérios estabelecidos, sendo a maior parte deles $(91,5 \%)$ no período diurno em que havia a presença de algum membro da equipe de pesquisa. 
Durante o período de internação na maternidade, foram realizados os seguintes procedimentos: seleção dos participantes do estudo, de acordo com os critérios de inclusão/exclusão explicitados; coleta de sangue das mães para dosagem de hemoglobina no pré-parto; e coleta de sangue do cordão umbilical para as dosagens de hemoglobina e de ferritina. Foram coletados $2 \mathrm{~mL}$ de sangue venoso das mães com scalp em tubo com EDTA K3 da marca Vacuette (Greiner Bio-one, Brasil). Após o clampeamento do cordão umbilical foram coletados $7 \mathrm{~mL}$ de sangue do cordão para análise de hemoglobina em tubos de $2 \mathrm{~mL}$ com EDTA K3 e, para a ferritina, em tubos de $5 \mathrm{~mL}$ com gel, ambos da marca Vacuette.

Realizaram-se entrevistas com as mães para obtenção de informações sobre as condições sócio-econômicas, história reprodutiva e dados sobre a gestação e parto, bem como consultas a prontuários para obtenção de dados sobre as condições de nascimento do recém-nascido.

A avaliação das crianças três meses após o nascimento foi realizada no ambulatório do próprio hospital mediante agendamento prévio feito pela equipe da pesquisa durante o período de internação na maternidade, reforçado próximo à avaliação por meio de aerograma e telefonema.

Nas consultas de seguimento, foram realizados os seguintes procedimentos: entrevista com as mães para obtenção de dados sobre alimentação, morbidade e administração de suplementos vitamínicos para a criança; tomada de medidas antropométricas da criança (peso e comprimento); punção sangüínea de $3 \mathrm{~mL}$ de sangue da criança, com scalp e microtubos, sendo $1 \mathrm{~mL}$ para a realização da dosagem de hemoglobina e $2 \mathrm{~mL}$ para a de ferritina.

A aplicação das entrevistas e a tomada das medidas antropométricas foram realizadas pela equipe de pesquisadores previamente treinados, sem conhecimento prévio do grupo ao qual a criança pertencia de acordo com o tipo de clampeamento. As crianças foram pesadas nuas, em balança pediátrica eletrônica da marca Filizola (modelo BP Baby, Brasil), com capacidade de $15 \mathrm{~kg}$ e precisão de $5 \mathrm{~g}$; o comprimento foi medido com estadiômetro horizontal de madeira, com fita métrica fixada na superfície, com precisão de $1 \mathrm{~mm}$, construído artesanalmente seguindo as recomendações do manual publicado pela Fundação Oswaldo Cruz. As medidas foram coletadas e registradas em duplicata sem arredondamentos, sendo a média calculada posteriormente. A punção de sangue venoso foi realizada por profissional da equipe do hospital.

Com o intuito de reduzir as perdas de seguimento, nos casos de não comparecimento à consulta no ambulatório, novo agendamento foi feito por meio de contato telefônico e, no caso de nova falta, uma visita domiciliar foi agendada, na qual uma técnica de enfermagem, previamente treinada, realizou todos os procedimentos necessários.

As dosagens de ferritina foram feitas pelo método da quimioluminescência, automatizado no equipamento Immulite, modelo 2000 (DPC Medlab Produtos Médico-Hospitalares Ltda., Brasil) e as dosagens de hemoglobina pelo método automatizado no Equipamento Pentra, modelo 120 (Horiba Abx, França).

Os dados foram digitados no programa Epi Info 2002, o qual possui recursos que permitem a dupla entrada de dados para detectar possíveis erros de digitação. As análises estatísticas foram efetuadas no programa Stata 8.0 (Stata Corp., College Station, Estados Unidos).

Três casos do grupo de clampeamento tardio foram excluídos da análise por apresentarem valores de ferritina sérica superiores a três desviospadrão.

$\mathrm{O}$ efeito do clampeamento tardio sobre o perfil hematológico das crianças ajustado para variáveis de confusão foi analisado por meio de modelos de regressão linear múltiplos, específicos para hemoglobina e ferritina. Foram introduzidas nos modelos, além do tipo de clampeamento do cordão umbilical (variável explanatória), todas as variáveis que apresentaram valor de p inferior a 0,30 na comparação entre os dois grupos, permanecendo no modelo final as variáveis que alteraram em mais de $10 \%$ a magnitude do coeficiente de regressão da variável de interesse. Além disso, foram incluídas no modelo as seguintes variáveis: (a) ferririna do cordão umbilical - para controlar o efeito do momento inicial sobre os níveis de ferritina aos três meses de idade; (b) hemoglobina materna no pré-parto; (c) sexo; (d) idade da criança; (e) peso ao nascer, por serem apontadas na literatura como fatores de risco para anemia em lactentes; (f) uso de vitaminas/sulfato ferroso - para controle do aporte de ferro da criança; e (g) febre nos 15 dias que antecederam a consulta - para controlar o possível efeito de um processo inflamatório/infeccioso sobre o aumento da ferritina aos três meses de idade. O procedimento adotado para a modelagem foi o stepwise backward selection.

O estudo foi aprovado nos Comitês de Ética em Pesquisa do Hospital do Campo Limpo e do Instituto de Saúde, e está em consonância com a Resolução $n^{\circ}$. 196/96, do Conselho Nacional de Saúde.

Neste artigo serão apresentados os resultados da avaliação das crianças aos três meses de idade. 


\section{Resultados}

Dos 325 casos captados, 224 (68,9\%) crianças foram acompanhadas aos três meses de idade. As perdas ocorreram em função do não comparecimento das mães à consulta $(20,6 \%)$ ou perda de material (10,5\%). Assim, foram estudados 109 casos de clampeamento imediato e 115 de clampeamento tardio, conforme ilustra a Figura 1.

A Tabela 1 descreve as características das mães, de acordo com os aspectos sócio-econômicos, reprodutivos e específicos da última gestação, segundo o procedimento adotado no clampeamento do cordão umbilical.

Nos dois subgrupos, clampeamento imediato e tardio, os resultados revelaram mães jovens, com escolaridade média por volta de oitos anos de estudo, vivendo em sua maioria em domicílios com condições adequadas de saneamento básico e, cerca de um terço delas primigestas. $\mathrm{Na}$ gestação atual compareceram, em média, a sete consultas de pré-natal, em sua maioria não fumaram durante a gestação, fizeram uso de complexo vitamínico e sulfato ferroso, não fizeram tratamento para anemia, dando à luz, em média, com 39 semanas de gestação. No pré-natal e no pré-parto apresentaram níveis de hemoglobina por volta de $12,0 \mathrm{~g} / \mathrm{dL}$ e as prevalências de anemia observadas, embora superiores no grupo do

\section{Figura 1}

Fluxograma da amostra.

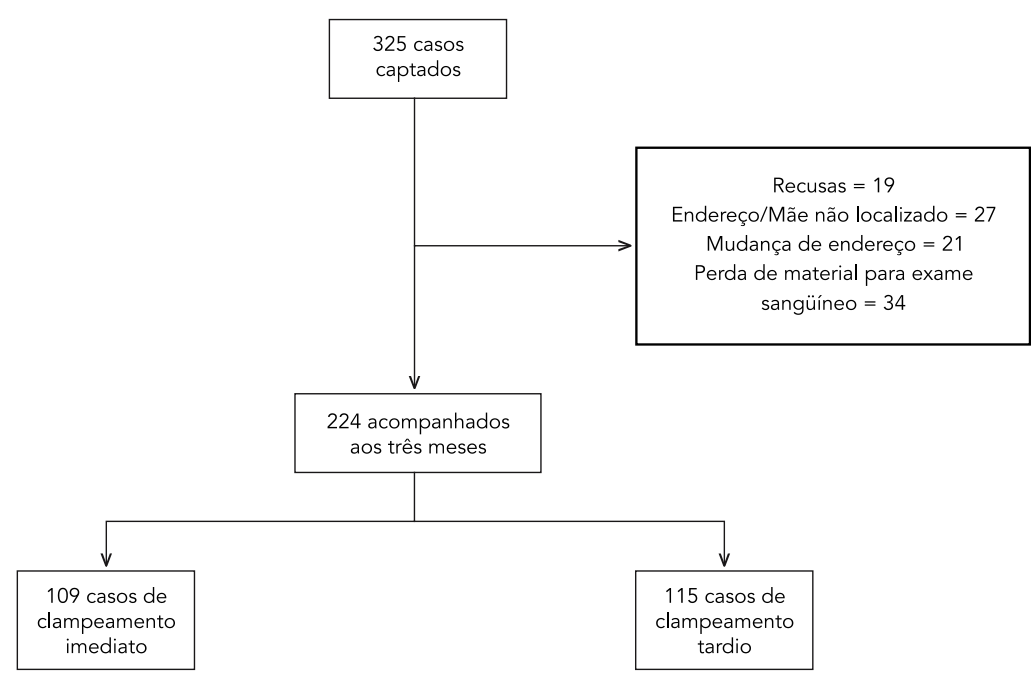

clampeamento imediato, não se mostraram estatisticamente diferentes do outro grupo.

Na Tabela 2 são apresentadas as características das crianças ao nascerem segundo tipo de clampeamento do cordão umbilical. A proporção de crianças do sexo masculino supera levemente a do feminino; apresentaram, em média, valores antropométricos e sinais vitais ao nascer adequados e semelhantes entre os grupos. Os valores médios de hemoglobina e ferritina ao nascimento não diferiram de acordo com o tipo de clampeamento do cordão umbilical.

Na Tabela 3 são apresentadas as características das mães e das crianças no acompanhamento ambulatorial após três meses do nascimento. A idade média das crianças nessa consulta foi de 3,22 meses ( $\mathrm{DP}=0,31$ ), em função de parte das mães não ter comparecido à consulta na data agendada.

Nesse período, as proporções relativas às intercorrências no pós-parto e à utilização de medicamentos pelas mães não se mostraram distintas entre os grupos. Com relação às crianças, observou-se semelhança no incremento de peso e comprimento; quanto aos níveis hematológicos, o valor médio de ferritina mostrouse superior no grupo de clampeamento tardio, embora a diferença não seja significativa; em relação à alimentação, os grupos diferiram quanto ao consumo de leite materno; a utilização de vitaminas/sulfato ferroso foi bastante elevada (cerca de $80 \%$ ) nos dois grupos e as proporções dos sinais de patologia infecciosa nos últimos 15 dias anteriores à consulta não diferiram entre os grupos.

O efeito do clampeamento tardio do cordão umbilical sobre os níveis de ferritina mostrou-se significativo ( $p=0,040)$, de modo que a intervenção realizada foi responsável por um incremento de 23,29ng/mL na média de ferritina aos três meses de idade, independente das demais variáveis controladas no modelo (Tabela 4).

Foram testadas as interações entre o tempo de clampeamento e o peso ao nascer e sexo, mas ambas não permaneceram no modelo por não apresentarem significância estatística.

O modelo de regressão para verificar o efeito do tipo de clampeamento do cordão umbilical sobre os níveis de hemoglobina foi testado. $\mathrm{O}$ mesmo não foi apresentado em virtude de que os níveis de hemoglobina aos três meses sofreram influência somente do tipo de alimentação da criança, mostrando-se superiores quando a criança estava em aleitamento materno nas 24 horas que antecederam a consulta $(\mathrm{p}=0,04)$, após o controle de possíveis variáveis de confusão. 
Características das mães, segundo o tipo de clampeamento do cordão umbilical. São Paulo, Brasil, 2006.

\begin{tabular}{|c|c|c|c|c|c|c|c|}
\hline \multirow[t]{3}{*}{ Características } & \multicolumn{6}{|c|}{ Tipo de clampeamento } & \multirow{3}{*}{$\begin{array}{l}\text { Valor } \\
\text { de } p \text { * }\end{array}$} \\
\hline & \multicolumn{3}{|c|}{ Imediato } & \multicolumn{3}{|c|}{ Tardio } & \\
\hline & $\%$ & Média & Erro padrão & $\%$ & Média & Erro padrão & \\
\hline \multicolumn{8}{|l|}{ Sócio-econômicas } \\
\hline Idade (anos) & & 24,87 & 0,59 & & 25,38 & 0,68 & 0,573 \\
\hline Escolaridade (anos) & & 8,22 & 0,28 & & 7,79 & 0,27 & 0,298 \\
\hline Densidade domiciliar & & 1,36 & 0,65 & & 1,38 & 0,64 & 0,798 \\
\hline Água tratada & 65,14 & & 4,58 & 66,96 & & 4,46 & 0,774 \\
\hline Rede pública de esgoto & 71,43 & & 4,43 & 78,18 & & 3,95 & 0,254 \\
\hline \multicolumn{8}{|l|}{ Reprodutivas } \\
\hline Primigesta & 38,53 & & 4,68 & 33,04 & & 4,46 & 0,394 \\
\hline Filhos $<5$ anos & 55,22 & & 6,12 & 54,67 & & 5,79 & 0,947 \\
\hline Amamentou o último filho & 86,49 & & 5,70 & 87,80 & & 5,17 & 0,862 \\
\hline \multicolumn{8}{|l|}{ Gestação atual } \\
\hline Número de consultas pré-natal & & 7,29 & 0,25 & & 6,97 & 0,22 & 0,326 \\
\hline Tabagismo & 21,30 & & 3,96 & 22,32 & & 3,95 & 0,854 \\
\hline Uso de complexo vitamínico & 87,16 & & 3,22 & 91,96 & & 2,58 & 0,242 \\
\hline Sem tratamento para anemia & 85,32 & & 3,04 & 87,50 & & 3,14 & 0,636 \\
\hline Idade gestacional (semanas) & & 39,32 & 0,10 & & 39,35 & 0,09 & 0,821 \\
\hline Hemoglobina no pré-natal (g/dL) & & 12,48 & 0,12 & & 12,53 & 0,10 & 0,729 \\
\hline Anemia no pré-natal & 7,37 & & 2,69 & 6,93 & & 2,54 & 0,905 \\
\hline Hemoglobina no pré-parto $(\mathrm{g} / \mathrm{dL})$ & & 12,10 & 0,14 & & 12,07 & 0,13 & 0,860 \\
\hline Anemia no pré-parto & 21,13 & & 4,02 & 18,87 & & 3,82 & 0,680 \\
\hline
\end{tabular}

* Teste t para as médias e qui-quadrado para as proporções.

\section{Discussão}

A prática do clampeamento imediato em nosso meio é oriunda de recomendações provenientes das áreas de obstetrícia e neonatologia, tendo como principal argumento a prevenção de icterícia e policitemia. Dessa forma, a cultura hospitalar acabou por preconizar o clampeamento precoce de forma rotineira. Atualmente, porém, essa recomendação vem sofrendo críticas, em função do seu potencial impacto sobre a saúde do recém-nascido 13,14,16.

Neste estudo optou-se por recomendar como clampeamento "tardio" aquele realizado um minuto após o nascimento, ao invés daquele realizado quando cessam os batimentos do cordão, considerando que $80 \%$ da transfusão placentária ocorrem no primeiro minuto 17 ; além disto, adotou-se como recomendação a colocação do recém-nascido no mesmo nível da placenta, para evitar a policitemia ${ }^{18}$. Ressalta-se que neste estudo não foram identificadas diferenças significativas em relação à presença de icterícia neonatal entre os grupos de clampeamento precoce e tardio (dados não apresentados).
Desde já, é importante considerar limitação do presente estudo o fato de não ter sido possível realizar a dosagem da proteína C-reativa (PCR), utilizada para o diagnóstico de processos inflamatórios de fase aguda. Embora o nível de ferritina seja considerado o melhor indicador de impacto de uma intervenção sobre os níveis de ferro, sua concentração pode aumentar na vigência de processos inflamatórios, sendo indicada a sua análise concomitante à PCR ${ }^{20}$. Na tentativa de minimizar o efeito desse mecanismo sobre os resultados do estudo optou-se por incluir no modelo multivariado a informação, referida pela mãe, sobre a ocorrência de febre na criança nos 15 dias que antecederam a consulta.

Também deve-se considerar que o número de perda no seguimento, superior ao esperado, poderia comprometer a qualidade dos resultados. No entanto, os valores de distribuição das variáveis relacionadas à escolaridade e níveis de hemoglobina da mãe e níveis de hemoglobina e ferritina do cordão umbilical do grupo ausente não diferiram do grupo acompanhado.

Os cuidados metodológicos adotados neste estudo garantiram a qualidade das informações e 
Características das crianças ao nascimento, segundo tipo de clampeamento do cordão umbilical. São Paulo, Brasil, 2006.

\begin{tabular}{|c|c|c|c|c|c|c|c|}
\hline \multirow[t]{3}{*}{ Características } & \multicolumn{6}{|c|}{ Tipo de clampeamento } & \multirow{3}{*}{$\begin{array}{l}\text { Valor } \\
\text { de } p \text { * }\end{array}$} \\
\hline & \multicolumn{3}{|c|}{ Imediato ( $n=109)$} & \multicolumn{3}{|c|}{ Tardio $(n=112)$} & \\
\hline & $\%$ & Média & Erro padrão & $\%$ & Média & Erro padrão & \\
\hline Sexo masculino & 52,29 & & & 54,46 & & & 0,746 \\
\hline Peso ao nascer (g) & & $3.218,46$ & 38,73 & & $3.203,75$ & 39,22 & 0,790 \\
\hline Comprimento ao nascer $(\mathrm{cm})$ & & 47,87 & 0,18 & & 47,75 & 0,19 & 0,640 \\
\hline Perímetro cefálico ao nascer $(\mathrm{cm})$ & & 34,19 & 0,14 & & 34,20 & 0,14 & 0,924 \\
\hline Apgar 1 minuto & & 8,41 & 0,70 & & 8,48 & 0,66 & 0,462 \\
\hline Apgar 5 mintutos & & 9,31 & 0,56 & & 9,34 & 0,48 & 0,704 \\
\hline Hemoglobina do cordão (g/dL) & & 14,76 & 0,21 & & 14,26 & 0,16 & 0,06 \\
\hline Ferritina do cordão (ng/mL) & & 162,45 & 12,02 & & 154,88 & 9,67 & 0,625 \\
\hline
\end{tabular}

* Teste t para as médias e qui-quadrado para proporção.

Características das mães e das crianças, após três meses do nascimento, segundo tipo de clampeamento do cordão umbilical, $2006 / 2007$.

\begin{tabular}{|c|c|c|c|c|c|c|c|}
\hline \multirow[t]{3}{*}{ Características } & \multicolumn{6}{|c|}{ Tipo de clampeamento } & \multirow{3}{*}{$\begin{array}{l}\text { Valor } \\
\text { de } p \text { * }\end{array}$} \\
\hline & \multicolumn{3}{|c|}{ Imediato } & \multicolumn{3}{|c|}{ Tardio } & \\
\hline & $\%$ & Média & Erro padrão & $\%$ & Média & Erro padrão & \\
\hline \multicolumn{8}{|l|}{ Mães (pós-parto) } \\
\hline Hemorragia & 2,75 & & 1,57 & 2,70 & & 1,55 & 0,982 \\
\hline Infecção & 5,5 & & 2,19 & 4,46 & & 1,96 & 0,722 \\
\hline Uso de vitamina & 70,09 & & 4,45 & 73,21 & & 4,20 & 0,608 \\
\hline Tratamento de anemia & 18,10 & & 3,78 & 14,41 & & 3,35 & 0,463 \\
\hline \multicolumn{8}{|l|}{ Crianças } \\
\hline Idade (meses) & & 3,234 & 0,317 & & 3,212 & 0,028 & 0,600 \\
\hline Peso (gramas) & & $6.106,86$ & 82,98 & & $6.063,70$ & 71,70 & 0,694 \\
\hline Comprimento $(\mathrm{cm})$ & & 60,47 & 0,25 & & 60,23 & 0,24 & 0,480 \\
\hline Hemoglobina (g/dL) & & 11,13 & 0,11 & & 11,18 & 0,09 & 0,730 \\
\hline Ferritina (ng/mL) & & 105,05 & 7,39 & & 123,15 & 9,92 & 0,146 \\
\hline Consumiu leite materno & 76,15 & & 4,10 & 88,39 & & 3,04 & 0,017 \\
\hline Aleitamento materno exclusivo & 34,86 & & 4,58 & 37,50 & & 4,59 & 0,683 \\
\hline Uso de vitaminas/sulfato ferroso & 77,78 & & 4,02 & 78,88 & & 3,92 & 0,914 \\
\hline Diarréia nos últimos 15 dias & 6,42 & & 2,36 & 3,60 & & 1,78 & 0,338 \\
\hline Febre nos últimos 15 dias & 11,01 & & 3,01 & 11,61 & & 3,04 & 0,888 \\
\hline Tosse nos últimos 15 dias & 33,03 & & 4,52 & 22,22 & & 3,95 & 0,075 \\
\hline
\end{tabular}

* Teste t para as médias e qui-quadrado para as proporções.

a possibilidade de discutirmos os achados frente aos resultados, ainda controversos, dos poucos estudos que tratam de avaliar os efeitos do clampeamento tardio sobre os níveis de hemoglobina e ferritina aos dois e três meses de idade, realizados em outros países.

O principal achado deste estudo revela que o clampeamento do cordão umbilical um minuto após o nascimento tem efeito positivo e independente sobre os níveis de ferritina aos três meses de vida. Esperava-se que o efeito da intervenção seria encontrado também em relação aos níveis de hemoglobina. No entanto, conforme verificado, os mesmos sofreram influência apenas do consumo de leite materno, o que pode ser atribuído à elevada biodisponibilidade do ferro 10 . 
Coeficientes de regressão relativos à associação entre níveis de ferritina das crianças aos três meses e tipo de clampeamento do cordão umbilical, ajustados para as variáveis de controle, 2006/2007.

\begin{tabular}{lccc}
\hline Variáveis & Coeficiente de regressão & IC95\% & Valor de $\mathbf{p}$ * \\
\hline Clampeamento tardio do cordão umbilical & 23,287 & $1,067-45,507$ & 0,040 \\
Ferritina do cordão umbilical & 0,336 & $0,236-0,437$ & 0,000 \\
Hemoglobina no pré-parto & $-6,066$ & $-14,360-2,228$ & 0,151 \\
Sexo & 45,461 & $22,947-67,974$ & 0,000 \\
Idade da criança & $-65,340$ & $-101,338-29,341$ & 0,000 \\
Peso ao nascer & 0,053 & $0,025-0,080$ & 0,000 \\
Febre nos 15 dias anteriores à consulta & $-38,473$ & $-74,362-2,584$ & 0,036 \\
Uso de vitaminas/sulfato ferroso & 10,466 & $-17,114-38,047$ & 0,455 \\
\hline
\end{tabular}

* Teste t para os coeficientes de regressão.

O efeito do tipo de clampeamento sobre os níveis de hemoglobina pode não ter sido observado pelo fato de que a carência de ferro ocorre no organismo de forma gradual e progressiva, sendo considerados três estágios até que a anemia se manifeste. O primeiro estágio, de depleção de ferro, afeta os depósitos do nutriente e representa um período de maior vulnerabilidade em relação ao seu balanço marginal, podendo progredir até uma deficiência mais grave. No segundo, de deficiência de ferro, ocorre eritropoiese ferro-deficiente e caracteriza-se por alterações bioquímicas que refletem a insuficiência de ferro para a produção normal da hemoglobina, ainda que a concentração de hemoglobina não esteja alterada. Somente no terceiro e último estágio ocorre diminuição dos níveis de hemoglobina, com repercussões funcionais 20,21 .

Estudo randomizado realizado na Guatemala evidenciou efeito positivo da intervenção sobre níveis de hemoglobina das crianças aos dois meses de vida. No entanto, apesar dos níveis de ferritina apresentarem-se superiores no grupo de clampeamento tardio quando comparado ao de clampeamento imediato não houve diferença significativa entre os grupos 18 .

Estudos sobre o clampeamento do cordão umbilical entre 1980 e 2001 foram revisados por Mercer 22, destacando-se como benefícios hematológicos elevados índices de hematocrito e ferritina para crianças a termo, aos dois meses de vida, que tiveram o cordão clampeado tardiamente; outros benefícios identificados incluem ausência de risco significativo de policitemia e hiperbilirrubinemia.

O efeito do clampeamento tardio do cordão umbilical sobre os níveis de ferritina e a ausência deste efeito nos níveis de hemoglobina, ain- da que aos seis meses de idade, também foram observados por Chaparro et al. 23 no México. Resultados de três estudos, conduzidos entre as décadas de 40 e 60, na África do Sul (Lanzkowsky, 1960, apud Chaparro et al. 23), China (PaoChen \& Tsu-Shan, 1960, apud Chaparro et al. 23) e Estados Unidos (Wilson et al., 1941, apud Chaparro et al. 23) não mostraram diferenças significativas nos níveis de hemoglobina entre crianças com clampeamentos precoce e tardio aos 3, 6 e 9 meses de idade.

Estudos de revisão apontam, entre as vantagens do retardamento do clampeamento do cordão umbilical, maior tempo para transferência do sangue presente na placenta no momento do nascimento, o que possibilita maior volume de sangue aos órgãos vitais na primeira semana de vida, podendo influenciar sobre o tempo de aleitamento materno, o que pode estar de acordo com os achados do presente estudo $(76 \%$ de consumo de leite materno no clampeamento imediato versus $88 \%$ no tardio $\mathrm{p}=0,017)$ 16,23.

van Rheenen \& Brabin 14 , em revisão da literatura, citam oito estudos randomizados que identificaram variáveis de confundimento que poderiam influenciar a relação entre o clampeamento do cordão umbilical e o perfil hematológico da criança. Concluíram que o clampeamento tardio proporciona aumento do estoque de ferro em crianças e, especialmente em filhos de mães anêmicas, aumenta a concentração de hemoglobina aos dois e três meses de idade, reduzindo o risco de anemia em crianças a termo.

No presente estudo, um dos possíveis fatores de confusão sobre a magnitude do impacto da intervenção poderia ser a freqüência do uso de vitaminas/sulfato ferroso na gestação e/ou entre as crianças, prescrito, ainda que de forma profilá- 
tica, em consultas de rotina nos diversos serviços de saúde. No entanto, a utilização de vitaminas/ sulfato ferroso não implicou em viés, uma vez que a freqüência do uso destes medicamentos foi elevada nos dois grupos de clampeamento do cordão umbilical.

$\mathrm{O}$ resultado encontrado neste estudo, em lactentes aos três meses de idade, sugere que o clampeamento tardio consiste em uma alternativa adicional de intervenção sustentável, de baixo custo e estratégia segura para integrar programas que visem à redução da deficiência de ferro e anemia em crianças, principalmente em países em desenvolvimento.

Estudos de seguimento com crianças em idade superior são necessários para confirmar a efetividade da intervenção para prevenção de anemia na primeira infância. Isso poderá ser analisado com os dados de seguimento obtidos neste estudo com crianças aos seis meses de vida.

\section{Resumo}

Este estudo verificou o efeito do clampeamento tardio (um minuto após o nascimento) do cordão umbilical sobre os niveis de hemoglobina e ferritina em crianças aos três meses de vida. Foram alocadas 325 mães e crianças, nascidas a termo, de parto vaginal e sem patologias (164 no grupo do clampeamento imediato e 161 no tardio) em um hospital do Município de São Paulo, Brasil, em 2006. Realizaram-se dosagens de hemoglobina da mãe no pré-parto e de hemoglobina e ferritina do cordão umbilical. As crianças (69\%) foram acompanhadas no ambulatório, após três meses, ocorrendo coleta de sangue venoso para dosagem de hemoglobina e ferritina. Variáveis sócio-econômicas, reprodutivas, antropométricas e de alimentação da criança foram estudadas. Utilizou-se a regressão linear múltipla para a análise dos dados. O efeito do clampeamento tardio do cordão umbilical foi significativo somente para os níveis de ferritina das crianças aos três meses de idade $(p=0,040)$, sendo superior em 23,29ng/mL quando comparado às crianças submetidas ao clampeamento imediato. O procedimento pode se constituir em uma estratégia para prevenir a deficiência de ferro em lactentes.

Epidemiologia Nutricional; Anemia; Deficiência de Ferro; Crianças; Cordão Umbilical

\section{Colaboradores}

S. I. Venâncio e S. R. D. M. Saldiva tiveram sua participação no planejamento do estudo, no trabalho de campo, análise dos dados e na redação do artigo. R. B. Levy participou no planejamento do estudo, no trabalho de campo, no gerenciamento e análise do banco de dados e na redação do artigo. L. Mondini participou no trabalho de campo, análise de dados e na redação do artigo. M. C. G. P. Alves participou no planejamento da amostra do estudo, análise dos dados e na redação do artigo. S. L. Leung participou no planejamento do trabalho de campo e revisão do artigo.

\section{Agradecimentos}

Ao Conselho Nacional de Desenvolvimento Científico e Tecnológico (CNPq; processo 505952/2004-1). Taís Izidio de Oliveira por sua contribuição no trabalho de campo, em especial no acompanhamento dos procedimentos de clampeamento do cordão umbilical; Honorina de Almeida pela contribuição no atendimento ambulatorial; Rui de Paiva por sua contribuição no atendimento ambulatorial; Luana Fiengo Tanaka pela contribuição na elaboração e processamento do banco de dados; Marina Aguiar Ferraresso por contribuir na elaboração e processamento do banco de dados. 


\section{Referências}

1. United Nations Children's Fund/United Nations University/World Health Organization. Iron deficiency anaemia, assessment, prevention and control - a guide for programme managers. Geneva: World Health Organization; 2001.

2. Nestel P. Proceedings: interventions for child survival. http://www.jsi.com/intl/omni/ironmain htm (acessado em Set/2001).

3. United Nations Children's Fund. Preventing iron deficiency in women and children: background and consensus on key technical issues and resources for advocacy, planning and implementing national programmes. New York: United Nations Children's Fund; 1998. (UNICEF Technical Workshop).

4. Department of Nutrition and Health Development, World Health Organization. Assessing the iron states of populations. Geneva: World Health Organization; 2004.

5. Mora JO, Mora LM. Deficiencias de micronutrientes en América Latina y el Caribe: anemia ferropriva. Geneva: Organización Panamericana de la Salud/Organización Mundial de la Salud; 1997.

6. Monteiro CA, Szarfarc SC, Mondini L. Tendência secular da anemia. Rev Saúde Pública 2000; 34: 62-72.

7. Osório MM, Lira PIC, Batista-Filho M, Ashworth A. Prevalence of anemia in children 6-59 months old in the state of Pernambuco, Brazil. Rev Panam Salud Pública 2001; 10:101-7.

8. Silva LSM, Giugliani ERJ, Rangel D, Aerts GC. Prevalência e determinantes de anemia em crianças de Porto Alegre, RS, Brasil. Rev Saúde Pública 2001 35:66-73.

9. Neuman NA, Tanaka OY, Szarfarc SC, Guimarães PRV, Victora CG. Prevalência e fatores de risco para anemia no Sul do Brasil. Rev Saúde Pública 2000; 34:56-63.

10. Ackré J. Alimentação infantil: bases fisiológicas. São Paulo: Organização Mundial da Saúde; 1994.

11. Gleason G, Scrimshaw NS. An overview of the functional significance of iron deficiency. In Kraemer K, Zimmermann MB, editors. Nutritional anemia. Basel: Sight and Life Press; 2007. p. 45-58.
12. Domellöf M, Lonnerdal B, Dewey KG, Cohen RJ, Rivera LL, Hernell O. Sex differences in iron status during infancy. Pediatrics 2002; 110:545-52.

13. van Rheenen PF, Brabin BJ. A practical approach to timing cord clamping in resource poor settings. BMJ 2006; 333:954-58.

14. van Rheenen PV, Brabin BJ. Late umbilical cordclamping as an intervention for reducing iron deficiency anaemia in term infants in developing and industrialized countries: a systematic review. An Trop Paediatr 2004; 24:3-16.

15. Zaborowski EL. Pesando e medindo em uma unidade de saúde. Rio de Janeiro: Fundação Oswaldo Cruz; 1997.

16. Schumann K, Solomons NY. Safety of interventions to reduce nutritional anemia. In: Kraemer K, Zimmermann MB, editors. Nutritional anemia. Basel: Sight and Life Press; 2007. p. 285-314.

17. Division of Reproductive Health, World Health Organization. Care of the umbilical cord: a review of the evidence. Geneva: World Health Organization; 1998. (Technical Support, 98.4).

18. Grajeda R, Pérez-Escamilla R, Dewey KG. Delayed clamping of the umbilical cord improves hematologic status of Guatemalan infants at 2 mo of age. Am J Clin Nut 1997; 65:425-31.

19. Thurnham DI, Northrop-Clewes CA. Infection and the etiology of anemia. In: Kraemer K, Zimmermann MB, editors. Nutritional anemia. Basel: Sight and Life Press; 2007. p. 231-56.

20. Paiva AA, Rondó PHC, Guerra-Shinohara EM. Parâmetros para avaliação do estado nutricional de ferro. Rev Saúde Pública 2004; 34:21-6.

21. Beard JL, Dawson H, Piñero DJ. Iron metabolism: comprehensive review. Nutr Rev 1996; 54:295-317.

22. Mercer JS. Current best evidence: a review of the literature on umbilical cord camping. J Midwifery Womens Health 2001; 46:402-15.

23. Chaparro CM, Neufeld LM, Alavez GT, Cedillo REL, Dewey KG. Effect of timing of umbilical cord clamping on iron status in Mexican infants: a randomised controlled trial. Lancet 2006; 367:19972004.

Recebido em 08/Ago/2007

Versão final reapresentada em 13/Fev/2008

Aprovado em 26/Fev/2008 